\title{
Current treatment strategy for newly diagnosed chordoma of the mobile spine and sacrum: results of an international survey
}

\author{
Nicolas Dea, MD, MSc, FRCSC, ${ }^{1}$ Charles G. Fisher, MD, MHSc, FRCSC, ${ }^{1}$ Jeremy J. Reynolds, FRCS, ${ }^{2}$ \\ Joseph H. Schwab, MD, ${ }^{3}$ Laurence D. Rhines, MD, ${ }^{4}$ Ziya L. Gokaslan, MD, ${ }^{5}$ \\ Chetan Bettegowda, MD, PhD, ${ }^{6}$ Arjun Sahgal, MD, Áron Lazáry, MD, PhD, ${ }^{8}$ \\ Alessandro Luzzati, MD, ${ }^{9}$ Stefano Boriani, MD, ${ }^{10}$ Alessandro Gasbarrini, MD, ${ }^{11}$ Ilya Laufer, MD, ${ }^{12}$ \\ Raphaële Charest-Morin, MD, FRCSC, ${ }^{13}$ Feng Wei, MD, ${ }^{14}$ William Teixeira, MD, ${ }^{15}$ \\ Niccole M. Germscheid, MSc, ${ }^{16}$ Francis J. Hornicek, MD, PhD, ${ }^{17}$ Thomas F. DeLaney, MD, ${ }^{18}$ \\ John H. Shin, MD, ${ }^{19}$ and the AOSpine Knowledge Forum Tumor \\ 1Division of Spine Surgery, Vancouver General Hospital and The University of British Columbia, Vancouver, British Columbia, \\ Canada; ${ }^{2}$ Oxford Spinal Unit, Oxford University Hospital, Oxford, United Kingdom; ${ }^{3}$ Department of Orthopaedics, Massachusetts \\ General Hospital, Boston, Massachusetts; ${ }^{2}$ Department of Neurosurgery, The University of Texas MD Anderson Cancer \\ Center, Houston, Texas; ${ }^{5}$ Department of Neurosurgery, The Warren Alpert Medical School of Brown University, Rhode Island \\ Hospital, and The Miriam Hospital, Providence, Rhode Island; ${ }^{6}$ Department of Neurosurgery, Johns Hopkins University School \\ of Medicine, Baltimore, Maryland; ' ${ }^{7}$ Department of Radiation Oncology, University of Toronto and Sunnybrook Odette Cancer \\ Centre, Toronto, Ontario, Canada; ${ }^{8}$ National Center for Spinal Disorders and Buda Health Center, Budapest, Hungary; ${ }^{9}$ Centro \\ Di Chirurgia Oncologia Ortopedica e Ricostruttiva del Rachide (CCOORR) and ${ }^{10} \mathrm{GSpine} 4$ Spine Surgery Division, IRCCS \\ Istituto Ortopedico Galeazzi, Milan, Italy; ${ }^{11}$ Unit of Oncologic and Degenerative Spine Surgery, Rizzoli Institute, Bologna, Italy; \\ ${ }^{12}$ Department of Neurological Surgery, Memorial Sloan Kettering Cancer Center, New York, New York; ${ }^{13}$ Centre Hospitalier \\ Universitaire de Québec, Québec City, Québec, Canada; ${ }^{14}$ Department of Orthopaedics, Peking University Third Hospital, \\ HaiDian District, Beijing, China; ${ }^{15}$ Department of Neurosurgery, Institute of Cancer of the State of São Paulo, São Paulo, \\ Brazil; ${ }^{16}$ Research Department, AOSpine International, Davos, Switzerland; ${ }^{17}$ Department of Orthopaedic Surgery, University of \\ California, Los Angeles Medical Center, Los Angeles, California; and Departments of ${ }^{18}$ Radiation Oncology and ${ }^{19}$ Neurosurgery, \\ Massachusetts General Hospital, Boston, Massachusetts
}

OBJECTIVE The purpose of this study was to investigate the spectrum of current treatment protocols for managing newly diagnosed chordoma of the mobile spine and sacrum.

METHODS A survey on the treatment of spinal chordoma was distributed electronically to members of the AOSpine Knowledge Forum Tumor, including neurosurgeons, orthopedic surgeons, and radiation oncologists from North America, South America, Europe, Asia, and Australia. Survey participants were pre-identified clinicians from centers with expertise in the treatment of spinal tumors. The suvey responses were analyzed using descriptive statistics.

RESULTS Thirty-nine of 43 (91\%) participants completed the survey. Most (80\%) indicated that they favor en bloc resection without preoperative neoadjuvant radiation therapy (RT) when en bloc resection is feasible with acceptable morbidity. The main area of disagreement was with the role of postoperative RT, where $41 \%$ preferred giving RT only if positive margins were achieved and 38\% preferred giving RT irrespective of margin status. When en bloc resection would result in significant morbidity, $33 \%$ preferred planned intralesional resection followed by RT, and $33 \%$ preferred giving neoadjuvant RT prior to surgery. In total, 8 treatment protocols were identified: 3 in which en bloc resection is feasible with acceptable morbidity and 5 in which en bloc resection would result in significant morbidity.

CONCLUSIONS The results confirm that there is treatment variability across centers worldwide for managing newly diagnosed chordoma of the mobile spine and sacrum. This information will be used to design an international prospective cohort study to determine the most appropriate treatment strategy for patients with spinal chordoma.

https://thejns.org/doi/abs/10.3171/2018.6.SPINE18362

KEYWORDS chordoma; mobile spine; sacrum; treatment strategy; radiation therapy; oncology

ABBREVIATIONS HRQOL = health-related quality of life; RT = radiation therapy.

SUBMITTED April 1, 2018. ACCEPTED June 21, 2018.

INCLUDE WHEN CITING Published online October 19, 2018; DOI: 10.3171/2018.6.SPINE18362. 
$\mathrm{C}$ URRENT evidence supports en bloc resection with wide margins as the best method to achieve disease-free survival for mobile spine and sacral chordomas. ${ }^{1,9}$ Because of the unique anatomical and functional characteristics of the spine, achieving wide or marginal margins is often difficult, even in the most experienced hands. ${ }^{1,21,26}$ Moreover, for chordomas located in the upper cervical spine and sacrum, the cost of achieving these margins from an adverse event and health-related qualityof-life (HRQOL) perspective is substantial. ${ }^{5,22,24,25}$

Considering the limited systemic therapy options ${ }^{23}$ adjunctive radiation therapy (RT) is often utilized to address microscopic disease and ultimately improve outcomes, especially when tumor margins are violated. ${ }^{18,20}$ In a recent systematic review, the highest rates of local control and overall survival were achieved with early adjuvant RT.18

The optimal RT regimen remains elusive, and there are no data comparing different RT modalities in the treatment of chordomas. Most cited studies come from singlecenter experiences, where treatment regimens are based on local experience, local protocols, and access to specific and costly technologies (i.e., proton, carbon-ion). Each of these specialized centers has been able to develop considerable expertise with the specific RT resources they have. Because chordomas are rare and highly variable, it is unlikely that a single- or several-center research model will ever result in definitive answers; ${ }^{8}$ a large international multicenter study is needed.

The primary objective of this study was to describe the different treatment strategies currently employed to treat chordoma of the mobile spine and sacrum. These results were subsequently used to design a prospective cohort study combining centers with similar management stratagems into distinct cohorts.

\section{Methods}

\section{Survey Design}

A 24-question survey (see Appendix) was designed by 2 investigators (N.D. and J.H. Shin). Feedback and pilot testing was conducted by 2 other authors (C.G.F. and J.J.R.). The survey was constructed to answer 2 main questions: what is your preferred treatment strategy for a newly diagnosed chordoma of the mobile spine and sacrum in which 1) an en bloc resection is feasible with acceptable morbidity or 2) en bloc resection would result in significant morbidity (high cervical, high sacral)? The meaning of "significant morbidity" differs from surgeon to surgeon and from patient to patient and is also based on the surgeon's experience and patient preferences. Accordingly, this was left to the discretion of the respondent. Four categorical options were provided: 1) neoadjuvant RT-surgical resection-postoperative RT; 2) en bloc resection-postoperative RT irrespective of surgical marginal status; 3) planned intralesional resection-postoperative RT; and 4) en bloc resection-no adjuvant RT if wide or marginal margins. Additional questions about the RT timing (i.e., neoadjuvant, adjuvant, both), modality (i.e., photons, protons, carbon-ion), availability, and dosage regimens were included. Participants were also asked about their willingness to participate in a prospec- tive cohort study on the topic. Open text fields were provided for comments.

\section{Participants and Survey Administration}

The survey was uploaded in a web-based survey tool (SurveyMonkey, https://surveymonkey.com) and distributed by email in November 2017 to the members of the AOSpine Knowledge Forum Tumor. AOSpine Knowledge Forum Tumor members are recognized leaders in the field of spinal oncology and are committed to the advancement of spinal oncology research. A total of 43 spinal surgeons (orthopedic surgeons and neurosurgeons) and radiation oncologists from various locations who had expertise and a known interest in spinal chordoma as well as access to different RT technologies were targeted. Specialists from North America, South America, Europe, Asia, and Australia were included. Two reminders were sent via email to surgeons who had been sent the initial survey participation request but did not respond.

\section{Analysis}

The data were collected and were then analyzed using descriptive statistics.

\section{Results}

Thirty-nine of 43 (91\%) oncology specialists, representing 31 clinics from 12 countries that span 5 continents, completed the survey (Table 1). Respondents were predominately surgeons: $22(56 \%)$ described themselves as spine surgeons, $4(10 \%)$ as orthopedic surgeons, and 10 (26\%) as neurosurgeons. Three (8\%) radiation oncologists also completed the survey. Four specialists did not complete the survey: 2 surgeons, 1 radiation oncologist, and 1 medical oncologist. Two (5\%) respondents treat more than 20 chordomas per year, $4(10 \%)$ between 16 and 20, 5 (13\%) between 11 and 15, $12(31 \%)$ between 5 and 10, and $16(41 \%)$ less than 5 per year.

\section{Question 1: What Is Your Preferred Treatment Strategy for a Newly Diagnosed Chordoma of the Spine When En Bloc Resection Is Feasible With Acceptable Morbidity?}

In this scenario, most respondents preferred an en bloc resection without preoperative neoadjuvant RT (79\%) (Fig. 1). The lack of consensus appeared when deciding on the role of postoperative adjuvant RT following en bloc resection: 38\% preferred giving adjuvant RT to every patient irrespective of margin status and $41 \%$ preferred giving RT only if there was a concern about positive margins. Only 1 respondent opted for a planned intralesional resection.

\section{Question 2: What Is Your Preferred Treatment Strategy for Newly Diagnosed Chordoma of the Spine When En Bloc Resection Would Result in Significant Morbidity (Upper Cervical, High Sacrectomy)?}

Thirty-three percent of respondents preferred a planned intralesional resection followed by RT, 33\% preferred to give neoadjuvant RT prior to surgery, and $23 \%$ preferred not to give adjuvant RT if wide or marginal margins were present after en bloc resection (Fig. 2). The primary difference between the 2 scenarios was the number of par- 
ticipants not attempting to treat the patient with an en bloc resection alone, and instead relying more on adjuvant (and neoadjuvant) therapies. In this scenario, more respondents preferred preoperative RT prior to surgery (33\%) compared to the first scenario (18\%). One center commented on an option of definitive RT treatment alone for high-risk patients whose condition was deemed either medically inoperable or operable with significant morbidity. This treatment option of RT alone was not included in the questionnaire.

The results of questions 1 and 2 were stratified and analyzed based on the caseload of each participant. High caseload was defined as treating more than 10 chordomas per year (11 respondents) and was referred to as the experienced group, and low caseload was treating 10 or fewer chordomas per year (28 respondents) and was referred to as the less-experienced group. Seventy-three percent of the experienced group preferred en bloc resection without any adjuvant RT if there were no concerns about margins when en bloc was feasible compared to only $29 \%$ in the less-experienced group. None of the experienced group respondents opted to give adjuvant RT irrespective of the marginal status compared to $54 \%$ in the less-experienced group. Lastly, $27 \%$ and $14 \%$ preferred neo-adjuvant RT in the experienced and less-experienced groups, respectively. The results also differed for when en bloc resection would result in significant morbidity (question 2). Thirty-six percent of the experienced group preferred en bloc resection without any adjuvant RT if there is no violation of the tumor margin as opposed to $18 \%$ in the less-experienced group. Intralesional resection followed by postoperative RT was preferred by only $27 \%$ of the experienced group compared to $36 \%$ of the less-experienced group.

When specifically asked, $67 \%$ of the whole cohort mentioned that the extent of surgery (marginal status) affected their postoperative treatment strategy without however influencing the RT modality chosen for most (67\%). All radiation oncologists included $\mathrm{RT}$ in the treatment plan, either pre- and postoperatively $(67 \%)$ or postoperatively only $(33 \%)$, irrespective of the marginal status.

Among those who usually give preoperative RT, 11\% waited 0 to 2 weeks prior to surgery, 33\% waited 3 to 4 weeks, $44 \%$ waited 5 to 8 weeks, and $11 \%$ waited more than 8 weeks. Similarly, when giving postoperative RT, $36 \%$ waited 0 to 4 weeks after surgery, $41 \%$ waited 5 to 8 weeks, $10 \%$ waited 9 to 12 weeks, and $13 \%$ waited more than 12 weeks.

Responses related to RT dosing regimens were incomplete and noninterpretable and were thus omitted from further analysis.

Most respondents restaged their cases after preoperative RT (79\%) and after surgery (77\%). The most preferred local staging modality was MRI (77\%), whereas systemic staging included CT of the chest, abdomen, and pelvis (77\%); PET scan (49\%); and MRI of the brain (26\%).

As for the RT modality, most use a combination that includes stereotactic body RT (SBRT) (49\%), protons (38\%), photons (36\%), and/or carbon-ion therapy (10\%). The most frequent combination was photons and protons. Lastly, $74 \%$ of respondents have no difficulty accessing proton therapy treatment facilities, and only $15 \%$ have access to carbon-ion therapy.
TABLE 1. Participating centers

\begin{tabular}{|c|c|c|c|}
\hline $\begin{array}{l}\text { Center } \\
\text { No. }\end{array}$ & Clinic/Hospital & City & Country \\
\hline 1 & Vancouver General Hospital & $\begin{array}{l}\text { Vancouver, } \\
\text { BC }\end{array}$ & Canada \\
\hline 2 & $\begin{array}{l}\text { Massachusetts General Hospi- } \\
\text { tal (MGH) }\end{array}$ & Boston, MA & USA \\
\hline 3 & MD Anderson Cancer Center & Houston, TX & USA \\
\hline 4 & $\begin{array}{l}\text { Rhode Island and The Miriam } \\
\text { Hospitals }\end{array}$ & Providence, RI & USA \\
\hline 5 & The Johns Hopkins Hospital & Baltimore, MD & USA \\
\hline 6 & Toronto Western & Toronto, ON & Canada \\
\hline 7 & Sunnybrook & Toronto, ON & Canada \\
\hline 8 & $\begin{array}{l}\text { National Center for Spinal } \\
\text { Disorders }\end{array}$ & Budapest & Hungary \\
\hline 9 & $\begin{array}{l}\text { IRCCS Galeazzi Orthopaedic } \\
\text { Institute }\end{array}$ & Milan & Italy \\
\hline 10 & Rizzoli Orthopaedic Institute & Bologna & Italy \\
\hline 11 & Oxford University Hospitals & Oxford & UK \\
\hline 12 & $\begin{array}{l}\text { Memorial Sloan Kettering } \\
\text { Cancer Center }\end{array}$ & New York, NY & USA \\
\hline 13 & Rush University Medical Center & Chicago, IL & USA \\
\hline 14 & Mayo Clinic & $\begin{array}{l}\text { Rochester, } \\
\text { MN }\end{array}$ & USA \\
\hline 15 & $\begin{array}{l}\text { University of Rochester Medi- } \\
\text { cal Center }\end{array}$ & Rochester, NY & USA \\
\hline 16 & Duke University Medical Center & Durham, NC & USA \\
\hline 17 & Peking University 3rd Hospital & Beijing & China \\
\hline 18 & $\begin{array}{l}\text { Institute of Cancer of São } \\
\text { Paulo (ICESP) }\end{array}$ & São Paulo, SP & Brazil \\
\hline 19 & $\begin{array}{l}\text { University Comprehensive } \\
\text { Spine Center Dresden }\end{array}$ & Dresden & Germany \\
\hline 20 & Monash Health & Melbourne & Australia \\
\hline 21 & Westchester Medical Center & Valhalla, NY & USA \\
\hline 22 & University Hospital & Basel & Switzerland \\
\hline 23 & UCLA Medical Center & $\begin{array}{l}\text { Los Angeles, } \\
\text { CA }\end{array}$ & USA \\
\hline 24 & $\begin{array}{l}\text { Vanderbilt University Medical } \\
\text { Center }\end{array}$ & Nashville, TN & USA \\
\hline 25 & $\begin{array}{l}\text { Hospital of the University of } \\
\text { Pennsylvania }\end{array}$ & $\begin{array}{l}\text { Philadelphia, } \\
\text { PA }\end{array}$ & USA \\
\hline 26 & UCSF Medical Center & $\begin{array}{l}\text { San Francis- } \\
\text { co, CA }\end{array}$ & USA \\
\hline 27 & Montreal General Hospital & Montreal, QC & Canada \\
\hline 28 & Hôpital de l'Enfant Jésus & $\begin{array}{l}\text { Quebec City, } \\
\text { QC }\end{array}$ & Canada \\
\hline 29 & $\begin{array}{l}\text { University Medical Center } \\
\text { Utrecht }\end{array}$ & Utrecht & $\begin{array}{l}\text { The Neth- } \\
\text { erlands }\end{array}$ \\
\hline 30 & National University Hospital & Singapore & Singapore \\
\hline 31 & $\begin{array}{l}\text { Orthopaedic University Hospi- } \\
\text { tal Friedrichsheim }\end{array}$ & Frankfurt & Germany \\
\hline
\end{tabular}

The centers shown in italic type (28-31) declined to participate in a prospective study. 


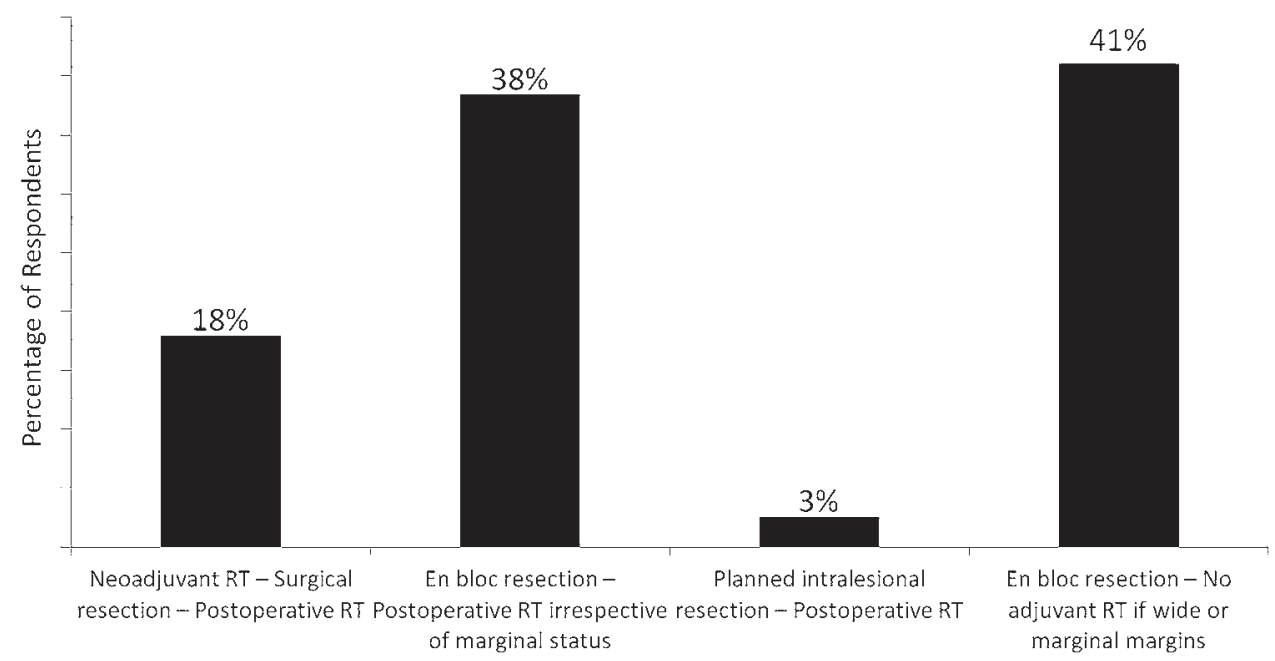

FIG. 1. Results for the first question: What is your preferred treatment strategy for a newly diagnosed chordoma of the spine when en bloc resection is feasible with acceptable morbidity?

Thirty-four of 39 respondents (87\%), representing 27 of 31 centers, expressed their willingness to participate in a multicenter prospective cohort study evaluating various treatment protocols for chordomas involving the mobile spine and sacrum. Most participants who declined to participate acknowledged that their tumor volume was too low to justify inclusion in such a study. Centers with similar treatment strategies were combined to form distinct treatment cohorts for a prospective study. Three treatment arms were identified for tumors for which en bloc resection is feasible with acceptable morbidity and 5 treatment arms were identified for tumors in which en bloc resection would result in significant morbidity (Fig. 3).

\section{Discussion}

The results of our survey illustrate the discrepancies and variation in the management of newly diagnosed spinal chordomas among some of the world's most experienced cancer centers. In this study, we show that for tumors that are amenable to en bloc resection with acceptable morbidity, it is unclear among our respondents whether RT should be given preoperatively, postoperatively, or at all, when margins respecting the Enneking classification are acheived. ${ }^{7}$ Considering the challenges of obtaining resections with wide margins, which according to Enneking would include the reactive zone outside the tumor, the majority of en bloc resections are marginal at best. In this scenario, the respondents were nearly evenly divided between whether to give postoperative RT or not. This represents 3 potential cohorts to study in our proposed multicenter prospective study: 1) patients who undergo preoperative RT followed by resection and postoperative RT, 2) patients who undergo en bloc resection followed by RT regardless

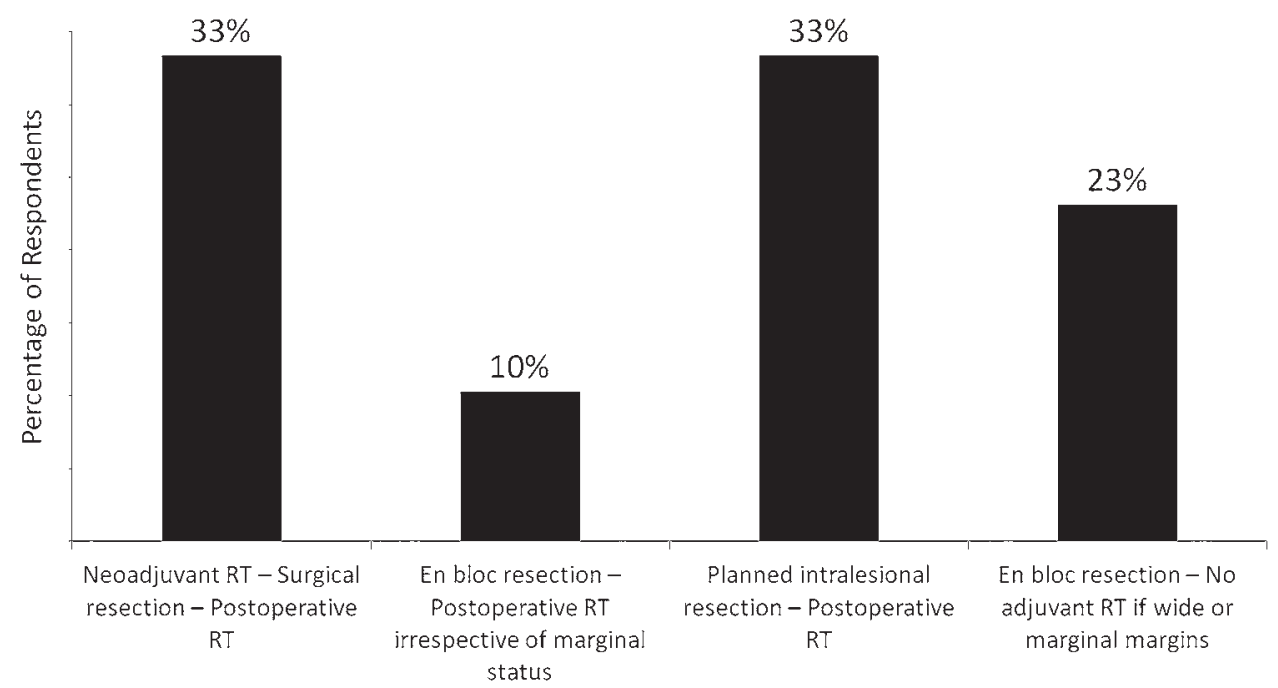

FIG. 2. Results for the second question: What is your preferred treatment strategy for newly diagnosed chordoma of the spine when en bloc resection would result in significant morbidity (upper cervical, high sacrectomy)? 


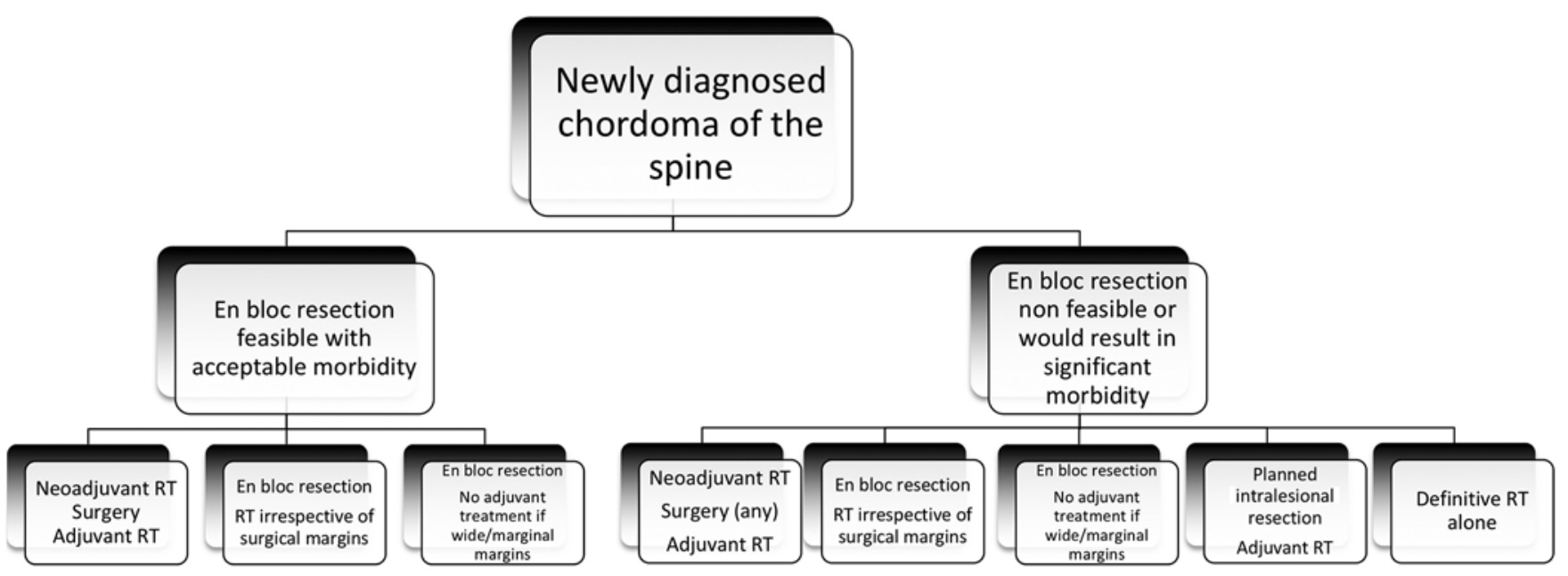

FIG. 3. Proposed treatment pathways for a prospective cohort study.

of margins, and 3) patients who undergo en bloc resection alone if margins are respected.

On the other hand, considering the morbidity and complications associated with treating tumors in difficult locations, there is understandably a push toward less invasive and less morbid treatment options for patients with these tumors. As demonstrated by this study, a significant proportion of participants would consider a deliberate intralesional resection or no surgery at all for these cases. This represents 2 additional cohorts to study in our proposed multicenter prospective study that differ from the other group. In difficult tumor locations thought to be excessively morbid for en bloc resection, what are the long-term local control rates with 1) intralesional resection and postoperative RT strategy and 2) RT alone? Whether or not these less surgically invasive options will result in durable long-term control is debatable, but the question is definitely worthy of investigation. More importantly, it needs to be determined whether the initial benefit in HRQOL conferred by a less-invasive treatment paradigm will be maintained in the long term. Likewise, the impact of such intralesional strategies on recurrence needs to be studied. ${ }^{3,5,15,27,28}$

Treatment of spinal chordoma is clearly evolving. Surgery plays a central role in the management of these tumors. The results of 2 recent systematic reviews have reiterated that achieving good surgical margins is associated with the best chances of local control and overall survival. ${ }^{2,18}$ Other chordoma-specific studies have reinforced these findings..$^{10,20,26}$ Questions regarding the role, type, timing, and prescription dose of RT remain unanswered. While it is accepted that high-dose conformal RT is necessary when there has been incomplete resection or when margins are concerning, ${ }^{2,18}$ there is no consensus in cases of Enneking-appropriate surgery. Because salvage surgery is rarely successful for patients with recurrent chordoma after initial surgery, some centers have preferred to use adjuvant postoperative RT, unless wide margins are achieved..$^{17}$ Moreover, some have reported that the best local control results are obtained with a strategy of neoadjuvant preoperative RT followed by en bloc resection and then postoperative RT boost. ${ }^{6,13,20}$ Early results with highdose single-fraction RT are also encouraging, ${ }^{29}$ and surprisingly good local control rates, similar to the best surgical series, are also being achieved with RT alone., , $12,14,16^{2}$

Although not our primary outcome, the results of this survey offer valuable insight into important questions such as timing of surgery and RT, staging techniques, and RT modality access. The timing of surgery after RT and vice versa is a common dilemma for the spine surgeon treating patients with metastatic disease. No literature is available on this topic in the primary tumor literature. Most of our survey respondents waited between 5 and 8 weeks before surgery after neoadjuvant RT and before adjuvant RT after surgery. Considering the devastating consequences of wound infection in this patient population, as well as the low early recurrence risk of spinal chordomas, it is sound to delay RT until after the wound is well healed. Moreover, complex flap reconstruction is often required in these cases, ${ }^{19}$ and it may potentially be impaired if the timing of surgery is too close to RT.

A limitation of this survey is that $92 \%$ of respondents were surgeons and only $8 \%$ were radiation oncologists. As a result, most RT regimen-specific questions were incompletely answered, and most respondents deferred to their radiation oncologist with respect to the dosage and fractionation questions. While the RT dosage is certainly critical for these tumors, we do not believe that the paucity of responses on that topic interfered with our primary survey goals. Those specific questions will be answered in the subsequent prospective study. This also limited comparative analyses between surgeons and radiation oncologists. Another limitation has to do with the fact that chordoma is a rare disease; even though we sent this survey to specialists who treat these tumors, $41 \%$ of them indicated that they treat less than 5 cases per year. Interestingly, oncology specialists who are treating more than 10 cases of chordoma per year favored a more aggressive surgical approach, even for tumors in difficult locations, and relied less on adjuvant therapy. The final limitation that we would like to note is that some respondents were trained by other respondents. This may lead to biases of having 
like-minded specialists respond in a similar way to the survey. To minimize this bias, we included as many different clinics as feasible from many regions of the world.

Chordomas have a low incidence and are pathologically heterogeneous, and as shown by this study, patients with these tumors may benefit from diverse treatment approaches. ${ }^{11}$ Optimal evidence-based care is lacking and is imperative. Knowledge gained from this study allowed us to design an international collaborative effort, which we hope will contribute significantly to answering these questions. Using this collaborative network of 27 different centers, we expect to be able to recruit a large volume of patients who will be included in predefined cohorts identified in the present study. Also, among the 27 participating sites, there is representation of various RT modalities, which will allow us to describe the local control rates and toxicities of the main RT options.

\section{Conclusions}

Treatment of spinal chordoma is multimodal and involves surgery and RT for most patients. Management options vary significantly across the world, due to resources, local expertise, and biases. Based on an international survey, 3 different treatment strategies were identified for chordoma of the spine that can be resected according to oncological principles with acceptable morbidity. Five treatment strategies are identified for spinal chordoma for which en bloc resection would result in significant morbidity. An international prospective cohort study involving 27 high-volume centers was designed based on the results of this survey.

\section{Acknowledgments}

This study was organized by AOSpine International, through the AOSpine Knowledge Forum Tumor, a pathology-focused working group of international spine experts acting on behalf of AOSpine in the domain of scientific expertise. We are especially grateful to the individuals who responded to the survey.

\section{References}

1. Boriani S, Bandiera S, Biagini R, Bacchini P, Boriani L, Cappuccio M, et al: Chordoma of the mobile spine: fifty years of experience. Spine (Phila Pa 1976) 31:493-503, 2006

2. Boriani S, Saravanja D, Yamada Y, Varga PP, Biagini R, Fisher CG: Challenges of local recurrence and cure in low grade malignant tumors of the spine. Spine (Phila Pa 1976) 34 (22 Suppl):S48-S57, 2009

3. Charest-Morin R, Dea N, Fisher CG: Health-related quality of life after spine surgery for primary bone tumour. Curr Treat Options Oncol 17:9, 2016

4. Chen YL, Liebsch N, Kobayashi W, Goldberg S, Kirsch D, Calkins G, et al: Definitive high-dose photon/proton radiotherapy for unresected mobile spine and sacral chordomas. Spine (Phila Pa 1976) 38:E930-E936, 2013

5. Dea N, Charest-Morin R, Sciubba DM, Bird JE, Disch AC, Mesfin A, et al: Optimizing the adverse event and HRQOL profiles in the management of primary spine tumors. Spine (Phila Pa 1976) 41 (Suppl 20):S212-S217, 2016

6. DeLaney TF, Liebsch NJ, Pedlow FX, Adams J, Weyman EA, Yeap BY, et al: Long-term results of Phase II study of high dose photon/proton radiotherapy in the management of spine chordomas, chondrosarcomas, and other sarcomas. J Surg Oncol 110:115-122, 2014
7. Enneking WF: A system of staging musculoskeletal neoplasms. Clin Orthop Relat Res (204):9-24, 1986

8. Fisher CG, Goldschlager T, Boriani S, Varga PP, Rhines LD, Fehlings MG, et al: An evidence-based medicine model for rare and often neglected neoplastic conditions. J Neurosurg Spine 21:704-710, 2014

9. Fuchs B, Dickey ID, Yaszemski MJ, Inwards CY, Sim FH: Operative management of sacral chordoma. J Bone Joint Surg Am 87:2211-2216, 2005

10. Gokaslan ZL, Zadnik PL, Sciubba DM, Germscheid N, Goodwin CR, Wolinsky JP, et al: Mobile spine chordoma: results of 166 patients from the AOSpine Knowledge Forum Tumor database. J Neurosurg Spine 24:644-651, 2016

11. Goodwin CR, Abu-Bonsrah N, Bilsky MH, Reynolds JJ, Rhines LD, Laufer I, et al: Clinical decision making: integrating advances in the molecular understanding of spine tumors. Spine (Phila Pa 1976) 41 (Suppl 20):S171-S177, 2016

12. Imai R, Kamada T, Tsuji H, Sugawara S, Serizawa I, Tsujii H, et al: Effect of carbon ion radiotherapy for sacral chordoma: results of Phase I-II and Phase II clinical trials. Int J Radiat Oncol Biol Phys 77:1470-1476, 2010

13. Indelicato DJ, Rotondo RL, Begosh-Mayne D, Scarborough MT, Gibbs CP, Morris CG, et al: A prospective outcomes study of proton therapy for chordomas and chondrosarcomas of the spine. Int J Radiat Oncol Biol Phys 95:297-303, 2016

14. Kabolizadeh P, Chen YL, Liebsch N, Hornicek FJ, Schwab $\mathrm{JH}$, Choy E, et al: Updated outcome and analysis of tumor response in mobile spine and sacral chordoma treated with definitive high-dose photon/proton radiation therapy. Int J Radiat Oncol Biol Phys 97:254-262, 2017

15. Molina CA, Ames CP, Chou D, Rhines LD, Hsieh PC, Zadnik PL, et al: Outcomes following attempted en bloc resection of cervical chordomas in the $\mathrm{C}-1$ and $\mathrm{C}-2$ region versus the subaxial region: a multiinstitutional experience. J Neurosurg Spine 21:348-356, 2014

16. Nishida Y, Kamada T, Imai R, Tsukushi S, Yamada Y, Sugiura H, et al: Clinical outcome of sacral chordoma with carbon ion radiotherapy compared with surgery. Int J Radiat Oncol Biol Phys 79:110-116, 2011

17. Park L, Delaney TF, Liebsch NJ, Hornicek FJ, Goldberg S, Mankin H, et al: Sacral chordomas: impact of high-dose proton/photon-beam radiation therapy combined with or without surgery for primary versus recurrent tumor. Int J Radiat Oncol Biol Phys 65:1514-1521, 2006

18. Pennicooke B, Laufer I, Sahgal A, Varga PP, Gokaslan ZL, Bilsky MH, et al: Safety and local control of radiation therapy for chordoma of the spine and sacrum: a systematic review. Spine (Phila Pa 1976) 41 (Suppl 20):S186-S192, 2016

19. Reynolds JJ, Khundkar R, Boriani S, Williams R, Rhines LD, Kawahara N, et al: Soft tissue and bone defect management in total sacrectomy for primary sacral tumors: a systematic review with expert recommendations. Spine (Phila Pa 1976) 41 (Suppl 20):S199-S204, 2016

20. Rotondo RL, Folkert W, Liebsch NJ, Chen YLE, Pedlow FX, Schwab JH, et al: High-dose proton-based radiation therapy in the management of spine chordomas: outcomes and clinicopathological prognostic factors. J Neurosurg Spine 23:788-797, 2015

21. Ruggieri P, Angelini A, Ussia G, Montalti M, Mercuri M: Surgical margins and local control in resection of sacral chordomas. Clin Orthop Relat Res 468:2939-2947, 2010

22. Schwab JH, Janssen SJ, Paulino Pereira NR, Chen YLE, Wain JC, DeLaney TF, et al: Quality of life after resection of a chordoma of the mobile spine. Bone Joint J 99-B:979-986, 2017

23. Stacchiotti S, Casali PG: Systemic therapy options for unresectable and metastatic chordomas. Curr Oncol Rep 13:323-330, 2011 
24. van Wulfften Palthe ODR, Houdek MT, Rose PS, Yaszemski MJ, Sim FH, Boland PJ, et al: How does the level of nerve root resection in en bloc sacrectomy influence patient-reported outcomes? Clin Orthop Relat Res 475:607-616, 2017

25. van Wulfften Palthe ODR, Janssen SJ, Wunder JS, Ferguson PC, Wei G, Rose PS, et al: What questionnaires to use when measuring quality of life in sacral tumor patients: the updated sacral tumor survey. Spine J 17:636-644, 2017

26. Varga PP, Szövérfi Z, Fisher CG, Boriani S, Gokaslan ZL, Dekutoski MB, et al: Surgical treatment of sacral chordoma: prognostic variables for local recurrence and overall survival. Eur Spine J 24:1092-1101, 2015

27. Verlaan JJ, Kuperus JS, Slooff WB, Hennipman A, Oner FC: Complications, secondary interventions and long term morbidity after en bloc sacrectomy. Eur Spine J 24:2209-2219, 2015

28. Wei F, Liu Z, Liu X, Jiang L, Dang G, Passias PG, et al: An approach to primary tumors of the upper cervical spine with spondylectomy using a combined approach: our experience with 19 cases. Spine (Phila Pa 1976) 43:81-88, 2018

29. Yamada Y, Laufer I, Cox BW, Lovelock DM, Maki RG, Zatcky JM, et al: Preliminary results of high-dose singlefraction radiotherapy for the management of chordomas of the spine and sacrum. Neurosurgery 73:673-680, 2013

\section{Disclosures}

Dr. Dea reports consultant relationships with Medtronic, Baxter, and Stryker and direct stock ownership in Medtronic. Dr. Fisher reports consultant relationships with Medtronic and NuVasive as well as receipt of royalties from Medtronic. Dr. Rhines reports an educational relationship with Stryker. Dr. Gokaslan reports direct stock ownership in Spinal Kinetics. Dr. Sahgal reports the follow- ing financial relationships: educational seminars with Elekta $A B$, Accuray Inc, and Varian Medical Systems; a research grant from Elekta AB; and travel accommodations/expenses from Elekta and Varian. Dr. Sahgal also reports belonging to the Elekta MR Linac Research Consortium. Dr. Laufer reports consultant relationships with Globus, Medtronic, DePuy/Synthes, Spinewave, and Brainlab. Ms. Germscheid reports an employee relationship with AOSpine International.

\section{Author Contributions}

Conception and design: Dea, Fisher, Reynolds, Shin. Acquisition of data: Dea, Germscheid, Shin. Analysis and interpretation of data: Dea, Germscheid, Shin. Drafting the article: Dea, Reynolds. Critically revising the article: all authors. Reviewed submitted version of manuscript: Dea, Fisher, Germscheid, Shin. Approved the final version of the manuscript on behalf of all authors: Dea. Statistical analysis: Dea, Germscheid, Shin. Administrative/technical/material support: Germscheid. Study supervision: Dea.

\section{Supplemental Information Online-Only Content}

Supplemental material is available with the online version of the article.

Appendix. https://thejns.org/doi/suppl/10.3171/2018.6.

\section{SPINE18362.}

\section{Correspondence}

Nicolas Dea: Vancouver General Hospital and The University of British Columbia, Vancouver, BC, Canada.nicolas.dea@vch.ca. 